ARTICLE

httpst//doi.org/10.1038/s414.67-020-20512-6

\title{
In situ NMR reveals real-time nanocrystal growth evolution via monomer-attachment or particle-coalescence
}

Reut Mashiach1, Haim Weissman, Liat Avram², Lothar Houben², Olga Brontvein², Anna Lavie ${ }^{3}$, Vaishali Arunachalam ${ }^{3}$, Michal Leskes (i) ${ }^{3}$, Boris Rybtchinski (i] ${ }^{1} \&$ Amnon Bar-Shir (i) ${ }^{1 \times}$

Understanding inorganic nanocrystal (NC) growth dynamic pathways under their native fabrication environment remains a central goal of science, as it is crucial for rationalizing novel nanoformulations with desired architectures and functionalities. We here present an insitu method for quantifying, in real time, NCs' size evolution at sub-nm resolution, their concentration, and reactants consumption rate for studying NC growth mechanisms. Analyzing sequential high-resolution liquid-state ${ }^{19} \mathrm{~F}-\mathrm{NMR}$ spectra obtained in-situ and validating by ex-situ cryoTEM, we explore the growth evolution of fluoride-based $\mathrm{NCs}\left(\mathrm{CaF}_{2}\right.$ and $\left.\mathrm{SrF}_{2}\right)$ in water, without disturbing the synthesis conditions. We find that the same nanomaterial $\left(\mathrm{CaF}_{2}\right)$ can grow by either a particle-coalescence or classical-growth mechanism, as regulated by the capping ligand, resulting in different crystallographic properties and functional features of the fabricated NC. The ability to reveal, in real time, mechanistic pathways at which NCs grow open unique opportunities for tunning the properties of functional materials.

\footnotetext{
${ }^{1}$ Department of Organic Chemistry, Weizmann Institute of Science, Rehovot 7610001, Israel. ${ }^{2}$ Department of Chemical Research Support, Weizmann Institute of Science, Rehovot 7610001, Israel. ${ }^{3}$ Department of Materials and Interfaces, Weizmann Institute of Science, Rehovot 7610001, Israel.

凶email: amnon.barshir@weizmann.ac.il
} 
C olloidal inorganic nanocrystals (NCs) have revolutionized science thanks to their provision of a controllable synthetic platform for tackling fundamental challenges in materials science and their utility in a variety of applications, from catalysis and renewable energy to nanomedicine ${ }^{1-6}$. Their exceptional features (e.g., optical, magnetic, chemical, catalytic, and electrical) often stem from their composition, size, and morphology, emphasizing the essentialness of mechanistic knowledge on NC formation from solution for strategizing novel fabrications. The relationships between NC growth pathways and resultant architectures and functions were revealed thanks to the development of a variety of ex situ and in situ methodologies ${ }^{7}$. Both spectroscopy- and microscopy-based techniques allow us to monitor NCs' size evolution, alternations in chemical composition, crystallography properties, and change in shape throughout the course of their formation $^{8-19}$. However, they often require temporal reaction sampling, destructive-energy radiation applications, the use of outof-equilibrium conditions (e.g., drying, freezing, heating, vacuum) and custom-made apparatuses. Thus, there is a need to implement accessible, alternative in situ approaches that can be performed under authentic nanofabrication settings.

Fluoride-based NCs (i.e., nanofluorides) have garnered much attention recently due to their potential use in optics, catalysis, optoelectronics, bioimaging, superconductor devices, batteries, and lubricants ${ }^{20}$. Amongst their advantages over other inorganic NCs, their low phonon energies and high chemical stability stand out, making nanofluorides one of the preferable host-materials for upconversion fluorescence ${ }^{21-26}$. These unique characteristics are governed by their crystallinity, size, shape, and introduced impurities, which are strictly regulated by their formation pathways $^{27-29}$. Interestingly, the rich fluoride content and relatively small-size of colloidal nanofluorides result in a characteristically high-resolution ${ }^{19} \mathrm{~F}-\mathrm{NMR}$ spectrum while in solution ${ }^{30}$. This property has been exploited to design water-dispersed $\mathrm{CaF}_{2} \mathrm{NCs}$ as nanotracers for in vivo ${ }^{19} \mathrm{~F}$-MRI applications, but also provides unprecedented opportunity to use nanofluorides as a platform for tracking NC formation in real time with a high-resolution (HR) NMR setup.

The unique capabilities of NMR-based approaches, which allow the monitoring of changes in the chemical environment of a variety of NMR active nuclei, offer a versatile tool for studying growth pathways and kinetics, surface chemistry, and the physical properties of both organic and inorganic materials ${ }^{31-36}$. In particular, the spectral resolution of NMR shows promise for the characterization of nanomaterials by way of detecting both the organic ligands $s^{37}$ and inorganic core elements ${ }^{38}$ as a means to explore their formation in solution. Moreover, the ability to spectrally resolve surface from bulk atoms via $\mathrm{NMR}^{39-43}$ makes this method an attractive way to characterize inorganic NC architecture.

Inspired by these studies, we herein present a high-resolution liquid-state NMR-based approach for studying the dynamics of NCs' size evolution in a quantitative manner without disturbing the reaction conditions (e.g., avoiding sampling, destructive radiation, unwanted heating, undesired freezing, drying, vacuum, etc.). By synthesizing nanofluorides under in situ NMR conditions, and based on the ability to detect the ${ }^{19} \mathrm{~F}-\mathrm{NMR}$ signals of $\mathrm{MF}_{2}\left(M=\mathrm{Ca}^{2}\right.$ + or $\left.\mathrm{Sr}^{2+}\right)$ in water, we are able to probe their sub-nm growth over the entire course of their formation, highlighting their controllable growth mechanisms (coalescence vs. classical simple grow), which result in different morphological and functional features.

\section{Results}

HR-NMR accurately determines the diameter of NCs in solution. Prior to conducting HR-NMR studies on water-dispersed, small-sized $\mathrm{CaF}_{2} \mathrm{NCs}$ capped with 2-aminoethyl phosphate (AEP, at pH7, molecular structure, Fig. 1a), they were synthesized and fully characterized with complementary techniques. CryoTEM images revealed monodispersed NCs with an average size of $3.7 \pm 0.6 \mathrm{~nm}$ (Fig. 1b and Supplementary Fig. 1a) with the confirmed composition of calcium, fluoride, and phosphate (from the ligand), as evaluated by high-angle annular dark-field (HAADF) STEM imaging and energy dispersive X-ray spectroscopy (EDS) (Fig. 1c). The fluorite, faced cubic centered (fcc), crystal structure of the obtained AEP-CaF 2 NCs was confirmed by powder X-ray diffraction (XRD, Supplementary Fig. 1b) and their relatively small size as well as their monodispersity were preserved in water, as revealed by dynamic light scattering (DLS, Supplementary Fig. 1c).

The high-resolution ${ }^{19} \mathrm{~F}-\mathrm{NMR}$ studies of the synthesized AEP$\mathrm{CaF}_{2} \mathrm{NCs}$ in water resulted in a ${ }^{19} \mathrm{~F}-\mathrm{NMR}$ spectrum, from which two populations of fluorides could be detected, at -105 and -109 ppm, assigned to fluorides at the surface and core of the NCs, respectively ${ }^{44,45}$ (Fig. 1d). This assignment was further supported by the significant paramagnetic effect on the ${ }^{19} \mathrm{~F}-\mathrm{NMR}$ peak attributed to the fluorides on the surface of the formulation (Supplementary Fig. 2) emphasizing one of the key features of NMR to provide detailed information that reflects the chemical environment of constituent nuclei. Indeed, NMR studies of nuclei within the NCs (rather than nuclei of the coating ligands) can distinguish between the nuclei at the NC's solid core and those located at the outer surface, due to differences in chemical environments, relaxation properties, and crystallinity characteristics ${ }^{39-43}$.

We next deconvolved the obtained ${ }^{19} \mathrm{~F}-\mathrm{NMR}$ spectrum for AEP-CaF ${ }_{2} \mathrm{NCs}$, using computational line fitting to quantify the ratio between the ${ }^{19} \mathrm{~F}$-atoms at the surface of $\mathrm{CaF}_{2} \mathrm{NCs}$ (Fig. 1d, pink) and the ${ }^{19} \mathrm{~F}$-atoms at the core of $\mathrm{CaF}_{2} \mathrm{NCs}$ (Fig. 1d, lightblue). From this ratio and by using Eq. (1) (Fig. 1e, Supplementary Note 1), we could calculate the average diameter of the AEP$\mathrm{CaF}_{2}$ NCs in water, which was found to be $4.1 \mathrm{~nm}$ (Fig. 1d). This ability to quantify the average diameter of water-dispersed nanofluorides from their HR- ${ }^{19}$ F-NMR spectrum by classification and quantification of surface and core atoms provides a unique approach for evaluating the average size of NCs while in solution.

To demonstrate the robustness and reproducibility of this approach, we applied it to evaluate the sizes of various nanofluoride $\left(\mathrm{CaF}_{2}\right.$ or $\left.\mathrm{SrF}_{2}\right)$ fabrications (Fig. If and Supplementary Fig. 3) from their HR ${ }^{19} \mathrm{~F}-\mathrm{NMR}$ spectra and found them to be in good correlation $\left(r^{2}=0.989\right)$ with the sizes obtained from HR-TEM images. This ability to accurately determine the average size of a variety of small-sized NCs demonstrates not only the uniqueness but also the potential variability of the proposed HRNMR-based method. Importantly, magic-angle spinning (MAS) solid-state ${ }^{19} \mathrm{~F}-\mathrm{NMR}$ of the same synthetic $\mathrm{AEP}-\mathrm{CaF}_{2}$ colloids' powder showed a similar ${ }^{19} \mathrm{~F}-\mathrm{NMR}$ spectrum (Supplementary Fig. 4a) to that obtained using high-resolution ${ }^{19} \mathrm{~F}-\mathrm{NMR}$ (Supplementary Fig. 4b), from which similar NC diameters were calculated. This observation confirms that water-dispersed AEP$\mathrm{CaF}_{2}$ NCs tumble fast enough in solution so as to average out the intrinsic homonuclear dipolar interactions, allowing to directly detect their fluoride content with $\mathrm{HR}$ liquid-state ${ }^{19} \mathrm{~F}-\mathrm{NMR}$ setups.

Observing NC growth in real time with HR-NMR. The ability to evaluate $\mathrm{NC}$ sizes at nanometer scale directly from solution without disturbing the system, using the HR 1D-NMR spectrum, offers a platform to monitor, in real time, NC growth dynamics. We validated this possibility by synthesizing nanofluorides in an NMR tube under ambient conditions while acquiring consecutive ${ }^{19} \mathrm{~F}-\mathrm{NMR}$ spectra from reaction initiation to final formation, 
a

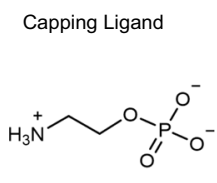

AEP

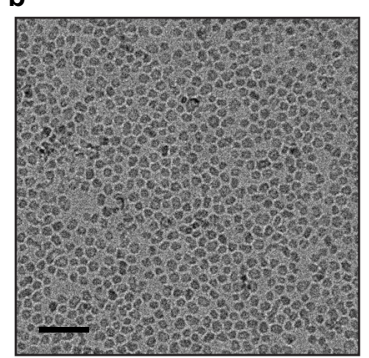

d

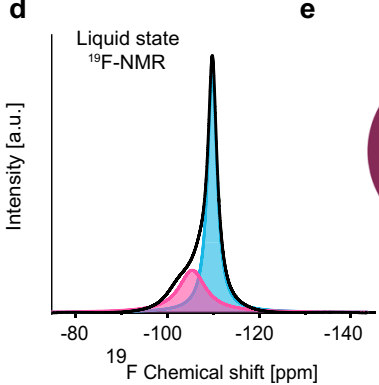

C

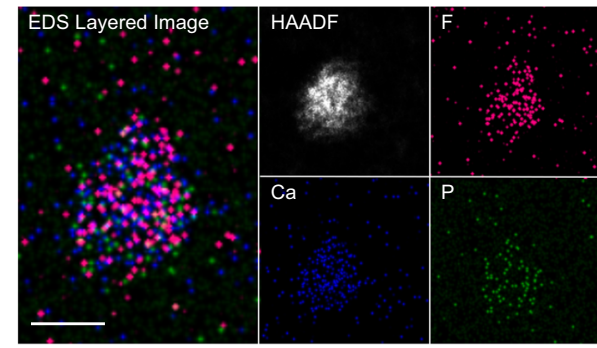

f

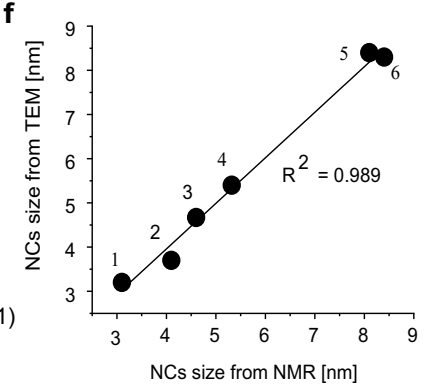

Fig. 1 Characterization of $\mathbf{A E P}-\mathbf{C a F}_{\mathbf{2}} \mathbf{N C s}$. a Molecular structure of an AEP ligand at pH 7. b Cryo-TEM AEP-CaF 2 NCs (scale bar 20 nm). c STEM-EDS elemental maps of an agglomerate of dried $\mathrm{AEP}-\mathrm{CaF}_{2} \mathrm{NCs}$ (scale bar $10 \mathrm{~nm}$ ). Shown are a combined map of the NCs elements, a high-angle annular darkfield (HAADF) image (white) and elemental maps of each element, i.e., F (pink), Ca (blue), and P (green). (d) Liquid-state, high-resolution 19F-NMR spectrum of water-dispersed AEP-CaF 2 NCs and its deconvoluted signals, attributed to ${ }^{19} \mathrm{~F}$ atoms at the surface of the NCs (-105 ppm, pink) and in the NC core (-109 ppm, light blue). e Schematic illustration of the $\mathrm{CaF}_{2} \mathrm{NC}$ with its surface (pink) and core atoms (light blue) and the equation (1) used to evaluate the size of NCs from the ${ }^{19} \mathrm{~F}-\mathrm{NMR}$ spectrum (Eq. (1) and Supplementary Note 1). $\mathbf{f}$ Correlation between the sizes measured with NMR and TEM of various $\mathrm{CaF}_{2}$ and $\mathrm{SrF}_{2} \mathrm{NCs}$ bearing different ligands (AEP, Citrate (Cit), Oleic Acid (OA) and Polyethylene Glycol (PEG)); (1) Cit-CaF 2 (Fig. 5b) (2) AEP$\mathrm{CaF}_{2}$ (3) AEP-SrF 2 (Fig. 3) (4) OA-SrF (Supplementary Fig. 3b) (5) PEG-CaF (Supplementary Fig. 3a) (6) OA-CaF 2 (Supplementary Fig. 3c).

monitoring simultaneously the size of the NCs, their concentration at each timepoint of the reaction and the consumption of the $\mathrm{F}^{-}$anions. Controlling the reaction conditions allowed to shed light on the dynamics of NC formation, highlighting their growth mechanism.

To synthesize the NCs, we started by adding a solution of $\mathrm{Ca}^{2+}$ cations to an aqueous reaction solution containing both $\mathrm{F}^{-}$anions and AEP capping ligands.

The NMR tube was then placed in a 9.4T NMR spectrometer and sequential ${ }^{19} \mathrm{~F}-\mathrm{NMR}$ spectra were acquired to follow the temporal formation of $\mathrm{CaF}_{2}$ colloids, resulting in a ${ }^{19} \mathrm{~F}-\mathrm{NMR}$ stack plot (Fig. 2a). Our results showed that each ${ }^{19} \mathrm{~F}-\mathrm{NMR}$ spectrum consisted of two main distinct peaks that could be, simultaneously, identified and assigned. One peak resonated at $-120 \mathrm{ppm}$, assigned to the free $\mathrm{F}^{-}$anion, indicating its consumption with time (Supplementary Fig. 5). The second downfield ${ }^{19} \mathrm{~F}-\mathrm{NMR}$ peak was attributed to the fluoride content of the $\mathrm{CaF}_{2} \mathrm{NC}$, from which the NC's diameter could be determined using the approach outlined in Fig. 1. Representative line fittings of the experimental ${ }^{19} \mathrm{~F}-\mathrm{NMR}$ spectra obtained at three different time points after reaction initiation (i.e., 6, 200, and $1000 \mathrm{~min}$ ) are presented in Fig. 2b, depicting the varying ratio between the two ${ }^{19} \mathrm{~F}$-spin populations of NCs, which reflects their size evolution. Representative cryo-TEM images of the reaction solution at the same reaction time points (i.e., 6, 200, and $1000 \mathrm{~min}$ ) are shown above each spectrum in Fig. $2 \mathrm{~b}$. The amounts of ${ }^{19} \mathrm{~F}$-atoms on the NC surface (pink) and in its core (light blue) were quantified and plotted as a function of the reaction time (Fig. 2c, 3 min temporal resolution). The diameter of the $\mathrm{CaF}_{2} \mathrm{NCs}$ at each timepoint was then derived from each individual ${ }^{19}$ F-NMR spectrum (using Eq. (1) and Supplementary Note 1) and plotted as a function of reaction time (Fig. 2d). From that plot, a notably rapid formation (3 min after reaction initiation) of ultrasmall $\mathrm{CaF}_{2} \mathrm{NCs}(2.3 \mathrm{~nm})$ is observed, followed by a period of relatively fast growth (up to $3.2 \mathrm{~nm}$ within the first $20 \mathrm{~min}$ ), which then gives way to much slower growth dynamics. In the regime of rapid NCs growth, the ${ }^{19} \mathrm{~F}-\mathrm{NMR}$ resonance of the surface atoms was significantly affected, as expected for smaller NCs that display a larger curvature (Supplementary Fig. 6).

Intriguingly, a mild but pronounced decrease in NC diameter was observed between 20 and $30 \mathrm{~min}$ (Fig. $2 \mathrm{~d}$ inset) that is followed by a continuous growth, a phenomenon that could indicate coalescence events during $\mathrm{NC}$ growth ${ }^{10,46,47}$. In parallel to the fast growth rate of the NCs (first 20 min, Fig. 2d), a significant reduction in their concentration was noted (first 20 min, Fig. 2e and Supplementary Equation 2), which was followed by a milder decrease in their total concentration. This observation that numerous small NCs transform into larger (but fewer) NCs, together with the detected intermediate stage (Fig. 2d inset), supports the notion that $\mathrm{AEP}-\mathrm{CaF}_{2} \mathrm{NCs}$ predominantly grow through coalescence ${ }^{7,48}$, a conclusion that is in good agreement with the "coalescence point" that can clearly be seen in the inset of Fig. $2 \mathrm{~d}$ and found to be reproducible $(N=4$, Supplementary Fig. 7). Such coalescence events were also captured by both cryoTEM (Supplementary Fig. 8) and high-resolution TEM (Supplementary Fig. 9) images sampled $25 \mathrm{~min}$ from the reaction initiation. Such a mechanistic pathway is promoted by the unfavorable thermodynamic state of initially formed ultrasmall $\mathrm{NCs}^{10}$, together with a relatively high number of NCs in solution, which may increase their collision probabilities and, essentially, lead to their coalescence to form larger, more stable NCs. Note here that Ostwald ripening mechanistic pathway may also occur but it is probably much less prominent than coalescence for AEP$\mathrm{CaF}_{2} \mathrm{NCs}$ growth, as reflected in both in situ NMR spectroscopy (Fig. 2 and Supplementary Fig. 7) and TEM imaging (Supplementary Fig. 8 and Supplementary Fig. 9). Importantly, we 
a

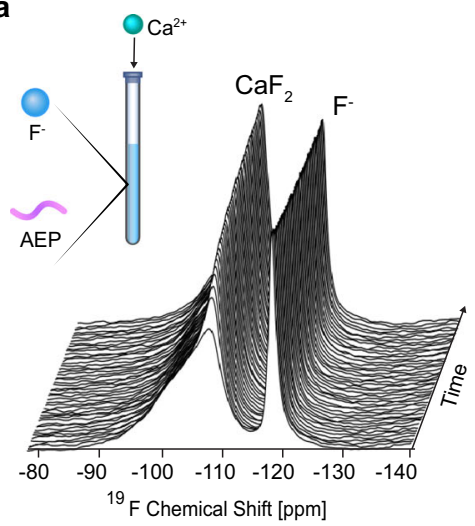

c

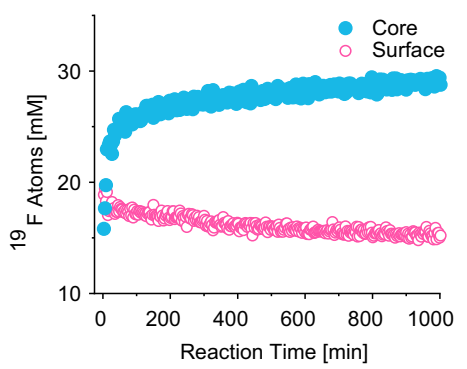

b

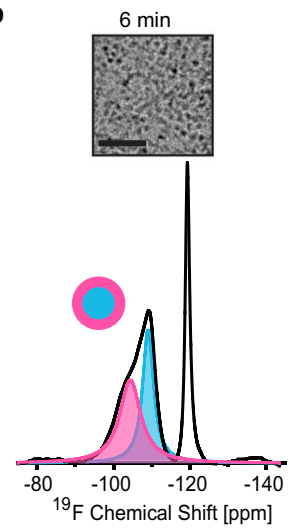

d

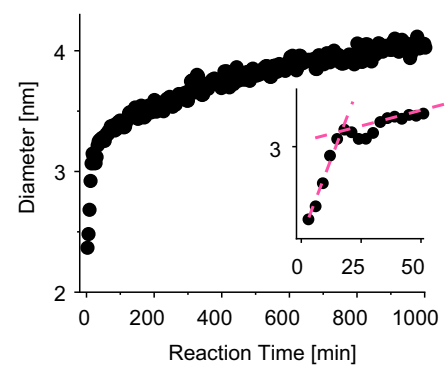

200 min
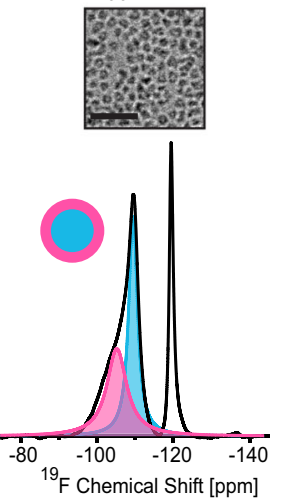

e

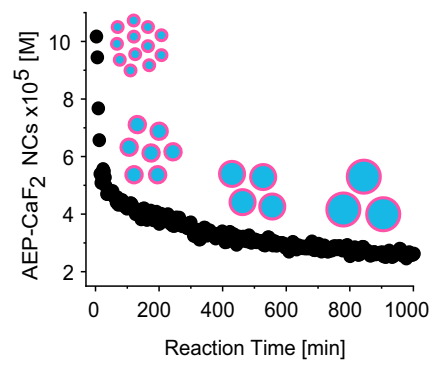

Fig. 2 Real-Time, high-resolution ${ }^{\mathbf{1 9}} \mathbf{F}-\mathbf{N M R}$ of $\mathbf{C a F}_{\mathbf{2}} \mathbf{N C}$ evolution in water. a Stack plot of real-time ${ }^{19} \mathrm{~F}$ NMR spectra after the addition of Ca+2 to the aqueous solution of AEP and $\mathrm{F}^{-}$anions. Shown spectra were taken every 30 min (experimental temporal resolution, 3 min). b Deconvoluted ${ }^{19} \mathrm{~F}-\mathrm{NMR}$ spectra from three different time points during the reaction $\left(6,200\right.$, and $1000 \mathrm{~min}$ ), showing the signals of ${ }^{19} \mathrm{~F}$ atoms on the surface (pink) and in the core (blue). Upper images are the correspondent cryo-TEM images of AEP-CaF 2 NCs. c Quantitative plot of the ${ }^{19} \mathrm{~F}-\mathrm{NMR}$ signals of the fluoride atoms in the core and on the surface of the formed NCs, calculated from the convoluted peaks' areas at -109 ppm and -105 ppm, respectively. $\mathbf{d}$ Evolution of the AEP$\mathrm{CaF}_{2} \mathrm{NC}$ diameter in water throughout the reaction process. Diameters were derived from Eq. (1). Inset shows the first 50 min of the reaction; dashed lines

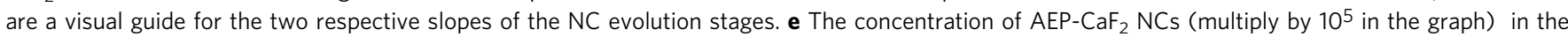
reaction solution as a function of reaction time, calculated from Supplementary Equation 2.

further validated the temporal change in the size of the NCs over time with ex situ cryo-TEM. To that end, the reaction mixture was sampled at three different stages (6 min, $200 \mathrm{~min}$, and 1000 min; Fig. 2b and Supplementary Fig. 10). The NC diameters obtained from cryo-TEM images (Supplementary Fig. 10e) were in a very good correlation (Supplementary Fig. $10 \mathrm{f}, R^{2}=0.98$ ) with those calculated from in situ ${ }^{19} \mathrm{~F}-\mathrm{NMR}$ at the same reaction stages.

The generality of the proposed in-situ ${ }^{19} \mathrm{~F}-\mathrm{NMR}$ for studying $\mathrm{NC}$ formation and growth was demonstrated also for $\mathrm{SrF}_{2} \mathrm{NCs}$ (Fig. 3 and Supplementary Fig. 11). Compared to $\mathrm{CaF}_{2} \mathrm{NCs}$, the $\mathrm{SrF}_{2} \mathrm{NCs}$ were larger, as determined by cryo-TEM (Fig. 3a, b) and DLS (Fig. 3c). The difference in size in the sub-nm range could be clearly depicted by the HR- ${ }^{19}$ F-NMR approach (Fig. 3d, e), and the method was applied to monitor, in situ and in real time, the formation of AEP-SrF 2 NCs. Notably, at three minutes after reaction initiation, the $\mathrm{SrF}_{2} \mathrm{NCs}$ were much larger than the $\mathrm{CaF}_{2}$ NCs $(2.9 \mathrm{~nm}$ vs. $2.3 \mathrm{~nm}$, respectively), a size difference that was preserved throughout the reaction. Similar to the case of $\mathrm{CaF}_{2}$, the temporal evolution of $\mathrm{SrF}_{2} \mathrm{NCs}$ is characterized by an initial fast growth followed by a moderate leveling-off of the size with time. Importantly, for both investigated nanofluorides, the concentration of the NCs in the solution decreased in parallel to their growth (Figs. 2d, e, 3e, and Supplementary Fig. 11b). To further elucidate this formation mechanism, we monitored the ligands during NC synthesis by way of in situ liquid-state ${ }^{31} \mathrm{P}-\mathrm{NMR}$ (Supplementary Fig. 12). The ${ }^{31} \mathrm{P}-\mathrm{NMR}$ peak of free AEP in solution is a clear narrow triplet. Upon the initial formation of
NCs, this triplet broadens probably due to the restricted mobility of the bound ligands to the numerous very small NCs that had formed. As the reaction progresses, when the number of NCs in the solution drops (Fig. 2e and Supplementary Fig. 11b), free AEP-ligands are re-released to the solution and their ${ }^{31} \mathrm{P}-\mathrm{NMR}$ peak narrows again due to their faster tumbling rates.

The role of ligands in the NC growth pathways. It is well known that ligands regulate NC formation mechanisms and dynamics, as they were found to be key elements in stabilizing both the reaction intermediates ${ }^{8,49}$ and formed colloids ${ }^{50,51}$. Specifically, differences in NC growth mechanisms have been attributed to the different affinities of the ligands to the cations $s^{52,53}$ and to their role in stabilizing the formed colloids ${ }^{54}$. Therefore, we decided to study the growth dynamics of $\mathrm{CaF}_{2} \mathrm{NCs}$ in water using a different capping ligand. In contrast to AEP (Fig. 1a), negatively charged ligands may hinder $\mathrm{NC}$ interactions at the NC's initial growth stage, due to electrostatic repulsion, which could lead to a different growth mechanism ${ }^{55}$. Interestingly, applying in situ ${ }^{19} \mathrm{~F}$ NMR to the synthesis of $\mathrm{CaF}_{2} \mathrm{NCs}$ in the presence of the negatively charged citric acid (citrate at $\mathrm{pH}=7$, Fig. $4 \mathrm{a}$ ) as the capping ligand revealed a different growth mechanism than that observed for AEP-CaF 2 (compare Fig. 2 and Fig. 4). We detected in the representative ${ }^{19} \mathrm{~F}-\mathrm{NMR}$ spectra obtained at different reaction time points (i.e., $6 \mathrm{~min}, 200 \mathrm{~min}$, and $1000 \mathrm{~min}$, Fig. 4b) a notable consumption of free fluoride (peak at $-120 \mathrm{ppm}$ ). This observation implies a growth mechanism that differs from particle- 
a

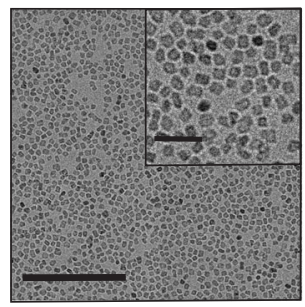

b
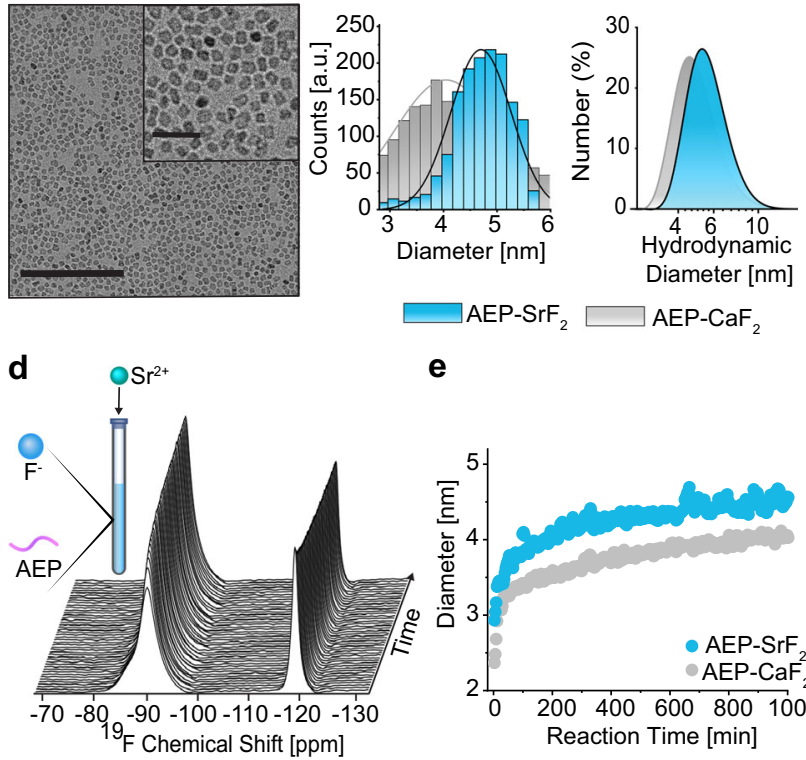

e

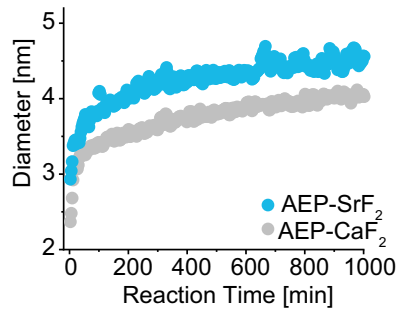

Fig. 3 AEP-SrF $\mathbf{2}$ NC formation. a Cryo-TEM of AEP-SrF 2 in water (1000 min after reaction initiation). b, c Size distributions of $\mathrm{AEP}-\mathrm{SrF}_{2}$ (light blue) and AEP-CaF 2 NCs (gray) as obtained from (b) cryo-TEM and (c) DLS. d Stack plot of the real-time ${ }^{19} \mathrm{~F}-\mathrm{NMR}$ spectra of AEP-SrF $\mathrm{F}_{2}$ synthesis. e Size evolution of the diameter of NCs in water throughout the reaction process for $\mathrm{AEP}-\mathrm{SrF}_{2}$ (light blue) and for $\mathrm{AEP}-\mathrm{CaF}_{2}$ (gray), as derived from Eq. (1). coalescence found for $\mathrm{AEP}-\mathrm{CaF}_{2}$. Assigning the ${ }^{19} \mathrm{~F}-\mathrm{NMR}$ peaks at -105 and -109 ppm to the fluorides of the surface and of the core of Cit- $\mathrm{CaF}_{2} \mathrm{NCs}$, respectively, allows to determine their size evolution (using Eq. (1) and Supplementary Note 1), revealing their continuous growth (Fig. 4c), while their final size was further verified by TEM (Supplementary Fig. 13).

Importantly, contrary to that observed for $\mathrm{AEP}-\mathrm{CaF}_{2} \mathrm{NCs}$ (Fig. 2e), the concentration of the growing Cit- $\mathrm{CaF}_{2} \mathrm{NCs}$ was near-constant throughout the synthesis (Fig. 4d, black). This observation, in addition to the significant depletion of the $\mathrm{F}^{-}$ anion throughout Cit- $\mathrm{CaF}_{2}$ 's growth (which is negligible for AEP$\mathrm{CaF}_{2}$, Supplementary Fig. 5), suggests a different growth mechanism. While AEP-CaF ${ }_{2}$ NCs grow mostly by coalescence, as reflected by the pronounced reduction in their concentration (Fig. 2), Cit-CaF $\mathrm{F}_{2} \mathrm{NCs}$ primarily grow by the classical simplegrowth mechanism ${ }^{10}$. This result emphasizes another advantage of our proposed approach. Besides its capability to determine NCs' size evolution, it can also differentiate between two different growth mechanisms as schematically depicted in Fig. 5 (coalescence vs. simple classical growth, Fig. 5a, b). Nevertheless, it is important to mention here that although the proposed in situ NMR approach allowed to investigate NC growth pathways, it failed to pick-up early and rapid nucleation phases.

Finally, we studied the crystallographic features and spin-lattice relaxation properties of $\mathrm{AEP}-\mathrm{CaF}_{2}$ and $\mathrm{Cit}-\mathrm{CaF}_{2} \mathrm{NCs}$, as it was shown that the growth mechanism as well as the properties of the capping ligands impact the obtained architectures and characteristics of as-synthesized $\mathrm{NCs}^{56,57}$. Interestingly, HR TEM images of the coalesced AEP-CaF $F_{2} \mathrm{NCs}$ showed crystallographic twinning defects for majority of the NCs (Fig. 5a and Supplementary Fig. 14), which were not observed in the crystalline Cit- $\mathrm{CaF}_{2} \mathrm{NCs}$ (Fig. 5b

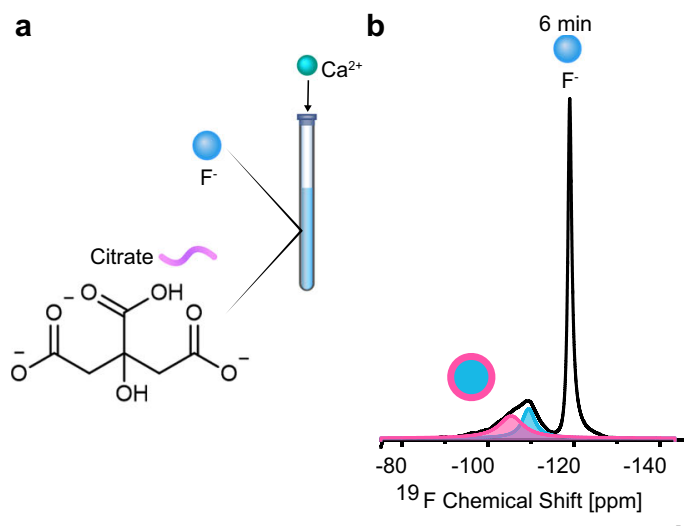

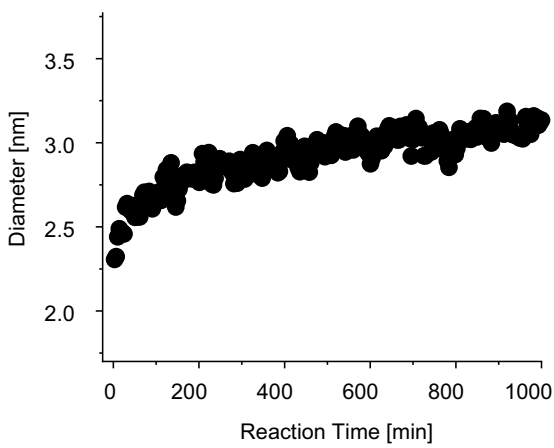

$200 \min$

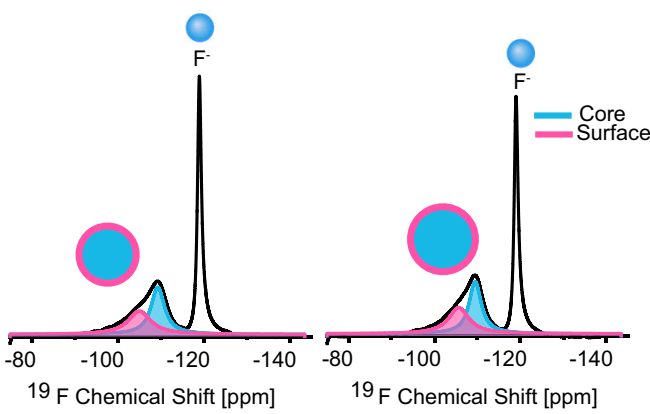

d

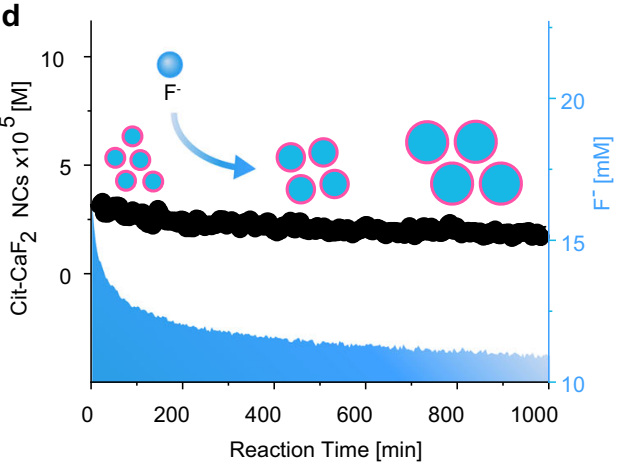

Fig. 4 Ligand effect on $\mathbf{C a F}_{\mathbf{2}} \mathbf{N C}$ 's growth mechanism. a Schematic illustration of the real-time synthesis of Cit-CaF $2 \mathrm{NCs}$ in water and of the molecular structure of citrate. $\mathbf{b}^{19} \mathrm{~F}-\mathrm{NMR}$ spectra from three different time points during the reaction ( $6 \mathrm{~min}, 200 \mathrm{~min}$, and $1000 \mathrm{~min}$ ), showing the deconvoluted

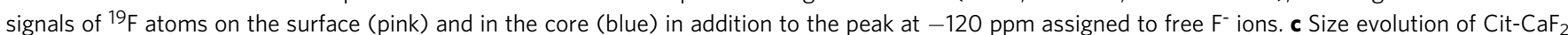
NCs in water from a real-time, high-resolution ${ }^{19} \mathrm{~F}-\mathrm{NMR}$ data set. $\mathbf{d}$ Concentration of Cit-CaF 2 NCs (multiply by 105 in the graph) in water (left axis, black) or $\mathrm{F}^{-}$ion consumption (right axis, light blue) as a function of the reaction time. 


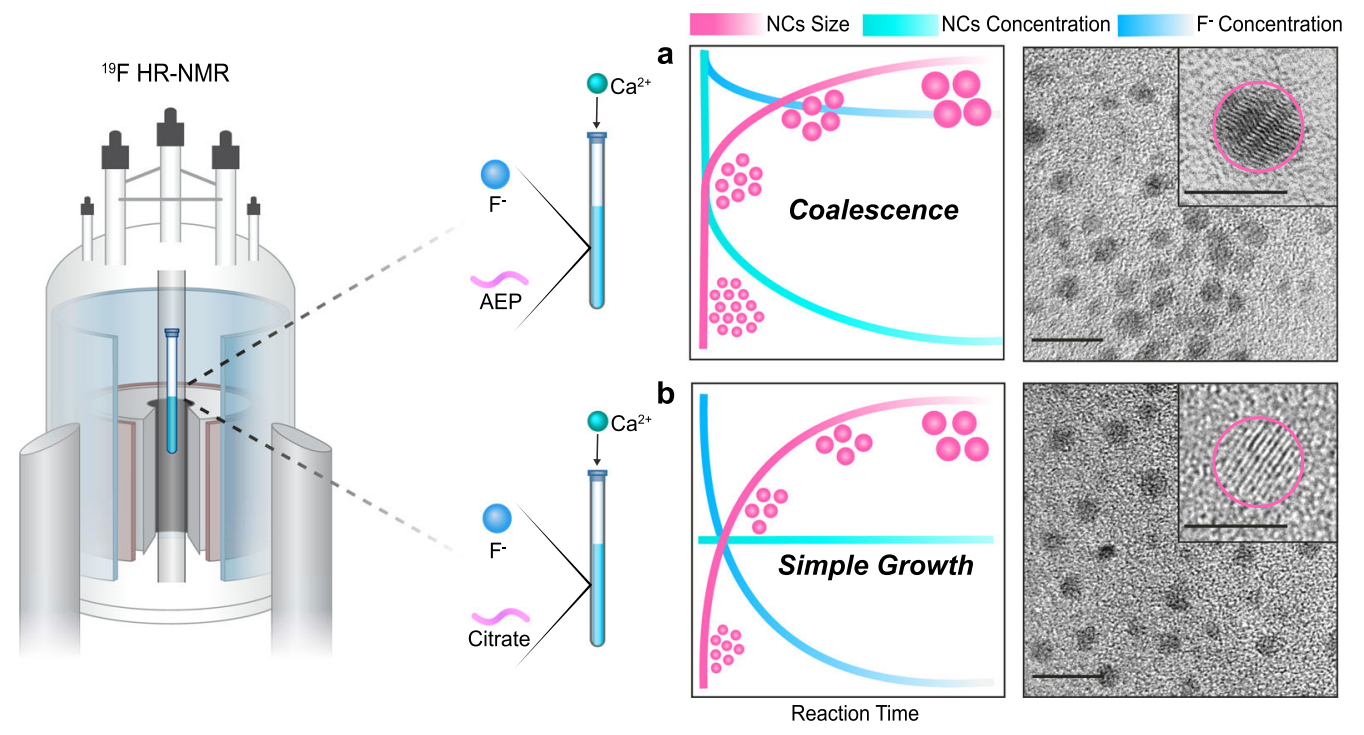

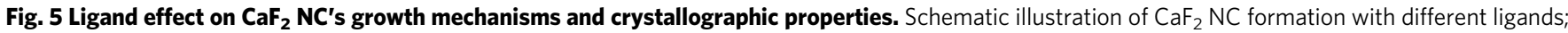
(a) AEP and (b) Citrate. The NCs' size (in pink), concentration (in turquoise) and $\mathrm{F}^{-}$ion concentration in solution (in blue). HR-TEM images of AEP-CaF 2 NCs presenting twinning defects (inset, in $\mathbf{a}$ ) and of $\mathrm{Cit}_{-} \mathrm{CaF}_{2} \mathrm{NCs}$ presenting a crystalline morphology (inset, in $\mathbf{b}$ ).

and Supplementary Fig. 14). In addition, the longitudinal relaxation time $\mathrm{T}_{1}$ of the NCs' fluoride content was shorter in AEP-CaF $\mathrm{F}_{2} \mathrm{NCs}$ as compared to that of Cit-CaF ${ }_{2}$ NCs (Supplementary Fig. 15). Such shorter $\mathrm{T}_{1}$ values of the defected NCs correlate with previous reports where mechanical stress-mediated lattice distortions of $\mathrm{CaF}_{2}$ powders resulted in significant reduced $\mathrm{T}_{1}$ values ${ }^{58}$, and should play a critical role in their future design as MRI tracers ${ }^{59}$. Our presented method for elucidating the contribution of the capping ligands to the nanofluoride's characteristics could be used for tuning them as functional nanomaterials for a variety of uses, as both their crystallinity ${ }^{60}$ and relaxation times ${ }^{59}$ were found to be key features in their performances.

In summary, we show here an approach for the real-time monitoring of NC evolution in solution based on the ability to detect in situ NMR signals of the elements of as-synthesized nanoformulations. The demonstrated ability to accurately determine the average NC diameter at each timepoint during the synthesis has provided us with valuable insight into NC growth mechanisms in solution. We found that in the presence of a phosphate-based ligand, NCs grow predominantly via particle coalescence, while a carboxylate-based ligand leads to a simple-growth mechanism of the same material. Each pathway yields different crystallographic features, and, consequently, distinct relaxation properties, highlighting the link between crystal growth dynamics, structure, and function. These findings should have numerous implications in the growing field of nanofluorides ${ }^{23-25,27,59,61}$ but also for other types of nanomaterials ${ }^{62-66}$ where the crystal properties determine the NC functionality. The generality of our NMR-based approach for revealing in-depth mechanistic insights of fluoride-NCs, is highlighted by the similar pattern of the NC evolution profiles found for other types of NCs studied by other in situ methods $10,46,47$. Therefore, we envision that the fundamental principles in NCs growth underlined here have the potential to serve as a foundation for future studies of other types of materials. Finally, the accessibility of high-resolution NMR spectrometers, in addition to the versatility of NMR acquisition schemes and the ability to study a variety of nuclei (beyond ${ }^{1} \mathrm{H}$ and ${ }^{19} \mathrm{~F}$ ), could be implemented to elucidate the growth mechanism from a perspective different from those provided by existing in situ techniques.

\section{Data availability}

The data that support the findings of this study are available from the corresponding author upon reasonable request.

Received: 3 April 2020; Accepted: 1 December 2020; Published online: 11 January 2021

\section{References}

1. Park, J. et al. Ultra-large-scale syntheses of monodisperse nanocrystals. Nat. Mater. 3, 891-895 (2004).

2. Alivisatos, $\mathrm{P}$. The use of nanocrystals in biological detection. Nat. Biotechnol. 22, 47-52 (2004).

3. Yu, J. H. et al. High-resolution three-photon biomedical imaging using doped ZnS nanocrystals. Nat. Mater. 12, 359-366 (2013).

4. Lee, J.-H. et al. Artificially engineered magnetic nanoparticles for ultrasensitive molecular imaging. Nat. Med. 13, 95 (2006).

5. Wang, D. \& Li, Y. Bimetallic nanocrystals: liquid-phase synthesis and catalytic applications. Adv. Mater. 23, 1044-1060 (2011).

6. Schaller, R. D. \& Klimov, V. I. High efficiency carrier multiplication in PbSe nanocrystals: implications for solar energy conversion. Phys. Rev. Lett. 92, 186601 (2004)

7. Thanh, N. T., Maclean, N. \& Mahiddine, S. Mechanisms of nucleation and growth of nanoparticles in solution. Chem. Rev. 114, 7610-7630 (2014).

8. Chang, H. et al. Molecular-level understanding of continuous growth from iron-oxo clusters to iron oxide nanoparticles. J. Am. Chem. Soc. 141, 7037-7045 (2019).

9. Li, J., Wang, H., Lin, L., Fang, Q. \& Peng, X. Quantitative identification of basic growth channels for formation of monodisperse nanocrystals. J. Am. Chem. Soc. 140, 5474-5484 (2018).

10. Zheng, H. et al. Observation of single colloidal platinum nanocrystal growth trajectories. Science 324, 1309-1312 (2009).

11. Cheong, S., Watt, J., Ingham, B., Toney, M. F. \& Tilley, R. D. In situ and ex situ studies of platinum nanocrystals: growth and evolution in solution. $J$. Am. Chem. Soc. 131, 14590-14595 (2009).

12. Williamson, M. J., Tromp, R. M., Vereecken, P. M., Hull, R. \& Ross, F. M. Dynamic microscopy of nanoscale cluster growth at the solid-liquid interface. Nat. Mater. 2, 532-536 (2003).

13. Peng, Z. A. \& Peng, X. Mechanisms of the shape evolution of CdSe nanocrystals. J. Am. Chem. Soc. 123, 1389-1395 (2001).

14. Polte, J. et al. Mechanism of gold nanoparticle formation in the classical citrate synthesis method derived from coupled in situ XANES and SAXS evaluation. J. Am. Chem. Soc. 132, 1296-1301 (2010).

15. Kwon, S. G. et al. Kinetics of monodisperse iron oxide nanocrystal formation by "heating-up" process. J. Am. Chem. Soc. 129, 12571-12584 (2007). 
16. Yuk, J. M. et al. High-resolution EM of colloidal nanocrystal growth using graphene liquid cells. Science 336, 61-64 (2012).

17. Kwon, O. et al. Exsolution trends and co-segregation aspects of self-grown catalyst nanoparticles in perovskites. Nat. Commun. 8, 15967 (2017).

18. Shen, X. et al. Deconvolution of octahedral Pt3Ni nanoparticle growth pathway from in situ characterizations. Nat. Commun. 9, 4485 (2018).

19. Mikhlin, Y. et al. Submicrometer intermediates in the citrate synthesis of gold nanoparticles: new insights into the nucleation and crystal growth mechanisms. J. Colloid Interface Sci. 362, 330-336 (2011).

20. Fedorov, P., Luginina, A., Kuznetsov, S. \& Osiko, V. Nanofluorides. J. Fluor. Chem. 132, 1012-1039 (2011).

21. Stouwdam, J. \& Veggel, F. Near-infrared emission of redispersible Er3+, Nd3 +, and Ho3+ doped LaF3 Nanoparticles. Nano Lett. 2, 733-737 (2002).

22. Heer, S., Kömpe, K., Güdel, H. U. \& Haase, M. Highly efficient multicolor upconversion emission in transparent colloids of lanthanide-doped NaYF4 nanocrystals. Adv. Mat. 16, 2102-2105 (2004).

23. Wang, F. \& Liu, X. Upconversion multicolor fine-tuning: visible to nearinfrared emission from lanthanide-doped NaYF4 nanoparticles. J. Am. Chem. Soc. 130, 5642-5643 (2008).

24. Zhao, J. et al. Single-nanocrystal sensitivity achieved by enhanced upconversion luminescence. Nat. Nanotechnol. 8, 729-734 (2013).

25. Liu, Y. et al. Amplified stimulated emission in upconversion nanoparticles for super-resolution nanoscopy. Nature 543, 229-233 (2017)

26. Deng, R. et al. Temporal full-colour tuning through non-steady-state upconversion. Nat. Nanotechnol. 10, 237-242 (2015).

27. Wang, F. et al. Simultaneous phase and size control of upconversion nanocrystals through lanthanide doping. Nature 463, 1061-1065 (2010).

28. Mai, H. X. et al. High-quality sodium rare-earth fluoride nanocrystals: controlled synthesis and optical properties. J. Am. Chem. Soc. 128, 6426-6436 (2006).

29. Chen, D. et al. Modifying the size and shape of monodisperse bifunctional alkaline-earth fluoride nanocrystals through lanthanide doping. J. Am. Chem. Soc. 132, 9976-9978 (2010).

30. Ashur, I., Allouche-Arnon, H. \& Bar-Shir, A. Calcium fluoride nanocrystals: tracers for in vivo 19F magnetic resonance imaging. Angew. Chem. Int. Ed. Engl. 57, 7478-7482 (2018).

31. Hughes, C. E., Williams, P. A. \& Harris, K. D. "CLASSIC NMR": an in-situ NMR strategy for mapping the time-evolution of crystallization processes by combined liquid-state and solid-state measurements. Angew. Chem. Int. Ed. Engl. 53, 8939-8943 (2014).

32. Goulet, P. J. \& Lennox, R. B. New insights into Brust-Schiffrin metal nanoparticle synthesis. J. Am. Chem. Soc. 132, 9582-9584 (2010).

33. Li, Y. et al. Mechanistic insights into the Brust-Schiffrin two-phase synthesis of organo-chalcogenate-protected metal nanoparticles. J. Am. Chem. Soc. 133, 2092-2095 (2011).

34. Doyen, M., Bartik, K. \& Bruylants, G. UV-Vis and NMR study of the formation of gold nanoparticles by citrate reduction: Observation of gold-citrate aggregates. J. Colloid Interface Sci. 399, 1-5 (2013).

35. Marbella, L. E. \& Millstone, J. E. NMR techniques for noble metal nanoparticles. Chem. Mater. 27, 2721-2739 (2015).

36. Vioglio, P. C. et al. A Strategy for probing the evolution of crystallization processes by low-temperature solid-state NMR and dynamic nuclear polarization. J. Phys. Chem. Lett. 10, 1505-1510 (2019).

37. Liu, X., Yu, M., Kim, H., Mameli, M. \& Stellacci, F. Determination of monolayer-protected gold nanoparticle ligand-shell morphology using NMR. Nat. Commun. 3, 1182 (2012).

38. Straney, P. J., Marbella, L. E., Andolina, C. M., Nuhfer, N. T. \& Millstone, J. E. Decoupling mechanisms of platinum deposition on colloidal gold nanoparticle substrates. J. Am. Chem. Soc. 136, 7873-7876 (2014).

39. Ladizhansky, V., Hodes, G. \& Vega, S. Surface properties of precipitated CdS nanoparticles studied by NMR. J. Phys. Chem. B 102, 8505-8509 (1998).

40. Rees, G. J. et al. Characterisation of platinum-based fuel cell catalyst materials using 195Pt wideline solid state NMR. Phys. Chem. Chem. Phys. 15, 17195-17207 (2013).

41. Thayer, A. M., Steigerwald, M. L., Duncan, T. M. \& Douglass, D. C. NMR study of semiconductor molecular clusters. Phys. Rev. Lett. 60, 2673-2676 (1988).

42. de Queiroz, T. B. et al. Probing surface effects on $\alpha$-NaYF4 nanoparticles by nuclear magnetic resonance. J. Phys. Chem. C. 124, 9523-9535 (2020).

43. Lovingood, D. D., Achey, R., Paravastu, A. K. \& Strouse, G. F. Size- and sitedependent reconstruction in CdSe QDs evidenced by $77 \mathrm{Se}\{1 \mathrm{H}\} \mathrm{CP}-\mathrm{MAS}$ NMR spectroscopy. J. Am. Chem. Soc. 132, 3344-3354 (2010).

44. Sadoc, A. et al. NMR parameters in alkali, alkaline earth and rare earth fluorides from first principle calculations. Phys. Chem. Chem. Phys. 13, 18539-18550 (2011).

45. Witter, R., Roming, M., Feldmann, C. \& Ulrich, A. S. Multilayered core-shell structure of polyol-stabilized calcium fluoride nanoparticles characterized by NMR. J. Colloid Interface Sci. 390, 250-257 (2013).
46. Niu, K.-Y., Liao, H.-G. \& Zheng, H. Visualization of the coalescence of bismuth nanoparticles. Microsc. Microanal. 20, 416-424 (2014).

47. Polte, J. et al. Nucleation and growth of gold nanoparticles studied via in situ small angle X-ray scattering at millisecond time resolution. ACS Nano 4 , 1076-1082 (2010)

48. Polte, J. Fundamental growth principles of colloidal metal nanoparticles-a new perspective. CrystEngComm 17, 6809-6830 (2015).

49. Boles, M. A., Ling, D., Hyeon, T. \& Talapin, D. V. The surface science of nanocrystals. Nat. Mater. 15, 141 (2016).

50. Nenon, D. P. et al. Design principles for trap-free $\mathrm{CsPbX} 3$ nanocrystals: enumerating and eliminating surface halide vacancies with softer Lewis bases. J. Am. Chem. Soc. 140, 17760-17772 (2018).

51. Lazzari, S. et al. Ligand-mediated nanocrystal growth. Langmuir 34, 3307-3315 (2018).

52. van Embden, J. \& Mulvaney, P. Nucleation and growth of CdSe nanocrystals in a binary ligand system. Langmuir 21, 10226-10233 (2005).

53. Owen, J. S., Chan, E. M., Liu, H. \& Alivisatos, A. P. Precursor conversion kinetics and the nucleation of cadmium selenide nanocrystals. J. Am. Chem. Soc. 132, 18206-18213 (2010).

54. Kovalenko, M. V., Scheele, M. \& Talapin, D. V. Colloidal nanocrystals with molecular metal chalcogenide surface ligands. Science 324, 1417-1420 (2009).

55. Heuer-Jungemann, A. et al. The role of ligands in the chemical synthesis and applications of inorganic nanoparticles. Chem. Rev. 119, 4819-4880 (2019).

56. Leite, E. et al. Crystal growth in colloidal tin oxide nanocrystals induced by coalescence at room temperature. Appl. Phys. Lett. 83, 1566-1568 (2003).

57. Penn, R. L. \& Banfield, J. F. J. S. Imperfect oriented attachment: dislocation generation in defect-free nanocrystals. Science 281, 969-971 (1998).

58. Abdellatief, M., Abele, M., Leoni, M. \& Scardi, P. Combined X-ray diffraction and solid-state $19 \mathrm{~F}$ magic angle spinning NMR analysis of lattice defects in nanocrystalline CaF2. J. Appl. Crystalogr. 46, 1049-1057 (2013).

59. Mashiach, R. et al. Inducing defects in 19F-nanocrystals provides paramagnetic-free relaxation enhancement for improved in vivo hotspot MRI Nano Lett. 20, 7207-7212 (2020).

60. Dyck, N. C., van Veggel, F. C. \& Demopoulos, G. P. Size-dependent maximization of upconversion efficiency of citrate-stabilized beta-phase NaYF4:Yb3+,Er3+ crystals via annealing. ACS Appl. Mater. Interfaces 5, 11661-11667 (2013)

61. Fan, X. et al. Defect-enriched iron fluoride-oxide nanoporous thin films bifunctional catalyst for water splitting. Nat. Commun. 9, 1809 (2018).

62. Bekenstein, Y. et al. The making and breaking of lead-free double perovskite nanocrystals of cesium silver-bismuth halide compositions. Nano Lett. 18, 3502-3508 (2018)

63. Kong, M. et al. Tuning the relative concentration ratio of bulk defects to surface defects in $\mathrm{TiO} 2$ nanocrystals leads to high photocatalytic efficiency. $J$. Am. Chem. Soc. 133, 16414-16417 (2011).

64. Hardy, D. A., Tigaa, R. A., McBride, J. R., Ortega, R. E. \& Strouse, G. F. Structure-fFunction correlation: engineering high quantum yields in downshifting nNanophosphors. J. Am. Chem. Soc. 141, 20416-20423 (2019).

65. Zhao, Z. et al. Octapod iron oxide nanoparticles as high-performance T2 contrast agents for magnetic resonance imaging. Nat. Commun. 4, 2266 (2013).

66. Mann, A. K. P., Wu, Z., Calaza, F. C. \& Overbury, S. H. Adsorption and reaction of acetaldehyde on shape-controlled $\mathrm{CeO} 2$ nanocrystals: elucidation of structure-function relationships. ACS Catal. 4, 2437-2448 (2014).

\section{Acknowledgements}

This project has received funding from the European Research Council (ERC) under the European Union's Horizon 2020 research and innovation program (grant agreement No 677715).

\section{Author contributions}

R.M. and A.B.-S. designed the study. R.M. and A.L. designed the $\mathrm{CaF}_{2}$ synthesis and R.M carried out the real-time synthesis of the nanofluorides. R.M. and L.A. designed and performed the high-resolution NMR experiments. R.M., V.A., and M.L. performed the computational line fitting of the NMR data. V.A. and M.L. performed the solid-state NMR experiments. H.W. and B.R. performed the cryo-TEM experiments. L.H. and O.B performed the EM-related studies (TEM and EDS). R.M. analyzed all the data shown and R.M. and A.B.-S. wrote the manuscript.

\section{Competing interests}

The authors declare no competing interests. 


\section{Additional information}

Supplementary information is available for this paper at https://doi.org/10.1038/s41467 020-20512-6.

Correspondence and requests for materials should be addressed to A.B.-S.

Peer review information Nature Communications thanks Jeffrey Reimer, Gilles Bruylants and the other, anonymous, reviewer(s) for their contribution to the peer review of this work.

Reprints and permission information is available at http://www.nature.com/reprints

Publisher's note Springer Nature remains neutral with regard to jurisdictional claims in published maps and institutional affiliations. (c) (i) Open Access This article is licensed under a Creative Commons Attribution 4.0 International License, which permits use, sharing, adaptation, distribution and reproduction in any medium or format, as long as you give appropriate credit to the original author(s) and the source, provide a link to the Creative Commons license, and indicate if changes were made. The images or other third party material in this article are included in the article's Creative Commons license, unless indicated otherwise in a credit line to the material. If material is not included in the article's Creative Commons license and your intended use is not permitted by statutory regulation or exceeds the permitted use, you will need to obtain permission directly from the copyright holder. To view a copy of this license, visit http://creativecommons.org/ licenses/by/4.0/.

(C) The Author(s) 2021 